\title{
Heat transfer analysis in wire bundles for aerospace vehicles
}

\author{
S. L. Rickman \& C. J. Iannello \\ NASA Engineering and Safety Center, USA
}

\begin{abstract}
Design of wiring for aerospace vehicles relies on an understanding of "ampacity" which refers to the current carrying capacity of wires, either, individually or in wire bundles. Designers rely on standards to derate allowable current flow to prevent exceedance of wire temperature limits due to resistive heat dissipation within the wires or wire bundles. These standards often add considerable margins and are based on empirical data. Commercial providers are taking an aggressive approach to wire sizing which challenges the conventional wisdom of the established standards. Thermal modelling of wire bundles may offer significant mass reduction in a system if the technique can be generalized to produce reliable temperature predictions for arbitrary bundle configurations. Thermal analysis has been applied to the problem of wire bundles wherein any or all of the wires within the bundle may carry current. Wire bundles present analytical challenges because the heat transfer path from conductors internal to the bundle is tortuous, relying on internal radiation and thermal interface conductance to move the heat from within the bundle to the external jacket where it can be carried away by convective and radiative heat transfer. The problem is further complicated by the dependence of wire electrical resistivity on temperature. Reduced heat transfers out of the bundle leads to higher conductor temperatures and, hence, increased resistive heat dissipation. Development of a generalized wire bundle thermal model is presented and compared with test data. The steady state heat balance for a single wire is derived and extended to the bundle configuration. The generalized model includes the effects of temperature varying resistance, internal radiation and thermal interface conductance, external radiation and temperature varying convective relief from the free surface. The sensitivity of the response to uncertainties in key model parameters is explored using Monte Carlo analysis.
\end{abstract}

Keywords: ampacity, cable harness, AS50881, wire bundle, thermal analysis. 


\section{Introduction}

Standard practice for cable derating within NASA programs has its roots in test data collected by the US military, the aviation industry, the Society for Automotive Engineers (SAE), and at NASA centers and traces back to the 1940s [1-3]. While these data were thoughtfully collected, documented and re-verified by subsequent studies, the resulting wire harness derating procedure still included significant conservatism [1-3]. There are many reasons for this added margin with the most significant contributor being the need to standardize a simple procedure that completely enveloped the envisioned use case and resulted in a capable design. Variables like total harness loading (i.e., load distribution), ambient temperature, cable construction, conductor alloys, insulation types, etc. are assumed to be consistent with the test conditions in the wire derating studies and formed the basis for the derating curves. Changes to these assumptions can have a significant effect on the ampacity of conductors within the harness allowing for larger margin than what is predicted by the curves. However, reclaiming this unaccounted for margin adds complexity to the analysis and added complexity generally runs counter to the derating procedure's primary goal which is safe design with an element of ease of use. In some of NASA's earliest references on standardizing wire harness design, the issue of a "one size fits all" wire harness derating was debated and lamented [1].

The problem of wire and wire bundle analysis has been studied by van Benthem et al. [4] and Ilgevičius and Liess [5]. Reference [4] focuses on thermal analysis of wire bundle configurations and highlights the complexity of the analysis by noting the numerous parameters involved. Reference [5] discusses thermal analysis of wires using a finite volume method and highlights the complexities involved with variable parameters and considered non-linear thermal conduction, convection and radiation as well as temperature varying electrical resistance in a single wire.

This paper seeks to address this longstanding issue via the use of a generalized model that considers key aspects of the harness design and use case that the current derating procedure does not fully consider such as insulation type and thickness, varying ambient temperatures, different conductor materials, various bundle loading configurations, mixed wire sizes and types in bundles, and more accurate vacuum prediction. Development of a single wire thermal model is presented and is extended to that of a bundle configuration. The model includes wire-to-wire heat conduction and radiation, the effect of temperature-varying resistance as well as external convection and radiation. The bundle model was easily implemented as a spreadsheet and can be used to solve steady state problems composed of up to fifty elements. A Monte Carlo capability has been implemented allowing exploration of problem sensitivity to, up to six variables.

\section{Development of the single wire thermal model}

A single powered wire terminated on both ends transfers heat to its surroundings through conduction along the wire to cooler terminals, conduction through the 
wire jacket and convection and radiation from the free surface. For steady state, the energy/time leaving the wire, $\dot{Q}_{\text {out }}$ through convection and radiation must equal the heat generation within the wire, $\dot{Q}_{g e n}$ :

$$
\dot{Q}_{\text {out }}=\dot{Q}_{\text {gen }} \text {. }
$$

For a sufficiently long wire, heat transfer from the terminations may be neglected and the energy/time leaving the wire is given by:

$$
\dot{Q}_{\text {out }}=\varepsilon A_{s} \sigma\left(T_{s}^{4}-T_{e}^{4}\right)+h A_{s}\left(T_{s}-T_{e}\right)
$$

where the wire surface area, $A_{s}$ is given by:

$$
A_{s}=2 \pi r_{s} L \text {. }
$$

First, assuming a constant resistance, heat generated within the wire through resistive dissipation is given by:

$$
\dot{Q}_{g e n}=I^{2} R \text {. }
$$

The resistance for a segment of wire of length, $L$ may be expressed as:

$$
R=R_{l} L .
$$

Combining the pertinent equations and simplifying yields the heat balance for the constant resistance case:

$$
I^{2} R_{l}=2 \pi r_{s}\left[\varepsilon \sigma\left(T_{s}^{4}-T_{e}^{4}\right)+f_{h} h\left(T_{s}-T_{e}\right)\right]
$$

where $f_{h}$ has been added as a scaling factor on convection and is zero for the vacuum case. For natural convection, the convective heat transfer coefficient, $h$ may be determined by noting the relationship to Nusselt number, $N u$ :

$$
N u=\frac{h\left(2 r_{s}\right)}{k}
$$

and the Nusselt number is a function of the Grashof-Prandtl number, GrPr:

$$
N u=f(G r P r)
$$

where...

$$
\operatorname{GrPr}=\frac{\rho^{2} \beta c_{p} g \Delta T\left(2 r_{s}\right)^{3}}{\mu k}
$$

$T_{\text {film }}$ is defined as:

$$
T_{\text {film }} \equiv \frac{T_{s}+T_{e}}{2} .
$$

Note that $\rho, c_{p}, \mu$, and $k$ for air are $f\left(T_{\text {film }}\right), \beta=\left(1 / T_{\text {film }}\right)$ and $\Delta T=\left(T_{s}-T_{e}\right)$.

The relationship between $\mathrm{Nu}$ and $\mathrm{GrPr}$ in eqn (8) is dependent upon the magnitude of $\mathrm{GrPr}$ and the geometry to be analyzed. For this analysis, a correlation presented in Holman [6] was used. Data extracted from the published plot were used to formulate a quadratic curve fit. Hence, the $N u$ was readily determined for a calculated $\mathrm{GrPr}$. 
The fluid properties are a function of the surface temperature, $T_{s}$ as well as the environment temperature which is assumed fixed. The convective heat transfer coefficient, $h=h\left(r_{s}, T_{s}\right)$.

In reality, electrical resistance varies with temperature and is expressed as:

$$
R=R_{0}\left[1+\alpha\left(T_{c}-T_{0}\right)\right]
$$

and the heat balance from eqn (6) becomes:

$$
I^{2} R_{l}\left[1+\alpha\left(T_{c}-T_{0}\right)\right]=2 \pi r_{s}\left[\varepsilon \sigma\left(T_{s}^{4}-T_{e}^{4}\right)+f_{h} h\left(r_{s}, T_{s}\right)\left(T_{s}-T_{e n v}\right)\right] .
$$

We note that the heat generated, represented by the left hand side of eqn (12) must be equal to the heat conducting out of the conductor into the insulation, or:

$$
I^{2} R_{l}\left[1+\alpha\left(T_{c}-T_{0}\right)\right]=\frac{2 \pi k_{w}\left(T_{c}-T_{s}\right)}{\ln \left(r_{s} / r_{c}\right)} .
$$

which leads to the solution for the conductor temperature:

$$
T_{c}=\frac{I^{2} R_{l}\left(\alpha T_{0}-1\right)-\frac{2 \pi k_{w} T_{s}}{\ln \left(r_{s} / r_{c}\right)}}{\alpha I^{2} R_{l}-\frac{2 \pi k_{w}}{\ln \left(r_{s} / r_{c}\right)}} .
$$

With some rearrangement and substitution, the overall heat balance becomes:

$$
\begin{aligned}
& I^{2} R_{l}\left\{1+\alpha\left[\frac{I^{2} R_{l}\left(\alpha T_{0}-1\right)-\frac{2 \pi k_{w} T_{s}}{\ln \left(r_{s} / r_{c}\right)}}{\alpha I^{2} R_{l}-\frac{2 \pi k_{w}}{\ln \left(r_{s} / r_{c}\right)}}-T_{0}\right]\right\} \\
& =2 \pi r_{s}\left[\varepsilon \sigma\left(T_{s}^{4}-T_{e}^{4}\right)+f_{h} h\left(r_{s}, T_{s}\right)\left(T_{s}-T_{e}\right)\right] .
\end{aligned}
$$

Once eqn (15) is solved iteratively for $T_{s}$, eqn (14) may be used to determine $T_{c}$.

\section{Development of the wire bundle thermal model}

The single wire heat balance may be extended to represent a collection of wires into a wire bundle. For the purpose of this analysis, it is assumed that adjacent conductors may transfer heat to one another via radiation and contact conductance. In this treatment, no internal convection is assumed. The collection of wires is assumed to be wrapped in an outer jacket which can exchange heat with the environment via convection and radiation. For model simplification, only conduction between the wires and the outer jacket is assumed.

The overall heat transfer between wires within the bundle is represented by the following system of equations: 


$$
\begin{aligned}
& \left(I^{2} R_{l}\right)_{i}\left\{1+\alpha_{i}\left[\left(T_{c}\right)_{i}-T_{0}\right]\right\} \\
& =\sum_{j=1}^{n} C_{i j}\left\{2 \pi\left(r_{s}\right)_{i} \varepsilon_{i} B_{i j} \sigma\left[\left(T_{s}^{4}\right)_{i}-\left(T_{s}^{4}\right)_{j}\right]\right. \\
& \left.+\left(G_{\text {int }}\right)_{i j}\left[\left(T_{s}\right)_{i}-\left(T_{s}\right)_{j}\right]\right\}
\end{aligned}
$$

where the factor $C_{i j}$ is unity when conductors $i$ and $j$ are adjacent to one another and zero otherwise. Note also that $C_{i j}=0$ when $i=j$. The radiation interchange factor, $B_{i j}$ is pre-computed using an external program for parallel cylinders of infinite extent in contact, spanning the range of radius ratios and surface optical properties and referenced as a look-up table as the model is formulated.

Ultimately, the solution to a system of linear equations is sought. However, the inclusion of thermal radiation poses a problem due to its highly non-linear nature. To resolve this problem, the radiation terms are linearized by noting:

$$
\begin{aligned}
& 2 \pi\left(r_{s}\right)_{i} \varepsilon_{i} B_{i j} \sigma\left[\left(T_{s}^{4}\right)_{i}-\left(T_{s}^{4}\right)_{j}\right] \\
& =2 \pi\left(r_{s}\right)_{i} \varepsilon_{i} B_{i j} \sigma\left[\left(T_{s}^{2}\right)_{i}+\left(T_{s}^{2}\right)_{j}\right]\left[\left(T_{s}\right)_{i}+\left(T_{s}\right)_{j}\right]\left[\left(T_{s}\right)_{i}-\left(T_{s}\right)_{j}\right] \\
& =\left(G_{\text {rad }}\right)_{i j}\left[\left(T_{s}\right)_{i}-\left(T_{s}\right)_{j}\right]
\end{aligned}
$$

where the linearized radiation conductor, $\left(G_{r a d}\right)_{i j}=2 \pi\left(r_{s}\right)_{i} \varepsilon_{i} B_{i j} \sigma\left[\left(T_{s}^{2}\right)_{i}+\right.$ $\left.\left(T_{s}^{2}\right)_{j}\right]\left[\left(T_{s}\right)_{i}+\left(T_{s}\right)_{j}\right]$. The overall heat balance becomes:

$$
\begin{aligned}
\left(I^{2} R_{l}\right)_{i}\left\{1+\alpha_{i}\left[\left(T_{c}\right)_{i}-T_{0}\right]\right\} & \\
= & \sum_{j=1}^{n} C_{i j}\left\{\left(G_{\text {rad }}\right)_{i j}\left[\left(T_{s}\right)_{i}-\left(T_{s}\right)_{j}\right]\right. \\
& \left.+\left(G_{\text {int }}\right)_{i j}\left[\left(T_{s}\right)_{i}-\left(T_{s}\right)_{j}\right]\right\} .
\end{aligned}
$$

For the bundle exterior surface, both convective and radiative relief are possible:

$$
\dot{Q}_{g e n}=2 \pi r_{b}\left[\varepsilon \sigma\left(T_{b}^{4}-T_{e}^{4}\right)+f_{h} h\left(r_{b}, T_{b}\right)\left(T_{b}-T_{e}\right)\right] .
$$

Finally, the linearized system of equations may be presented in compact form as:

$$
\left(I^{2} R_{l}\right)_{i}\left\{1+\alpha_{i}\left[\left(T_{c}\right)_{i}-T_{0}\right]\right\}=\sum_{j=1}^{n} C_{i j}\left(G_{e q}\right)_{i j}\left[\left(T_{s}\right)_{i}-\left(T_{s}\right)_{j}\right]
$$

where:

$$
\left(T_{c}\right)_{i}=\left[\frac{I^{2} R_{l}\left(\alpha T_{0}-1\right)-\frac{2 \pi k_{w} T_{s}}{\ln \left(r_{s} / r_{c}\right)}}{\alpha I^{2} R_{l}-\frac{2 \pi k_{w}}{\ln \left(r_{s} / r_{c}\right)}}\right]_{i}
$$

and...

$$
\left(G_{e q}\right)_{i j}=\left(G_{i n t}\right)_{i j}+\left(G_{r a d}\right)_{i j} .
$$

The overall system of linearized equations can be expressed in the form: 


$$
[G]\left\{T_{s}\right\}=\{\dot{Q}\} .
$$

Substituting the equations above into the matrix form yields the following:

$$
\left[\begin{array}{cccccc}
v_{11} & w_{12} & w_{13} & \cdots & w_{1 n} & w_{1 b} \\
w_{21} & v_{22} & w_{23} & \cdots & w_{2 n} & w_{2 b} \\
w_{31} & w_{32} & v_{33} & \cdots & w_{3 n} & w_{3 b} \\
\vdots & \vdots & \vdots & \ddots & \vdots & \vdots \\
w_{n 1} & w_{n 2} & w_{n 3} & \cdots & v_{n n} & w_{n b} \\
w_{b 1} & w_{b 2} & w_{b 3} & \cdots & w_{b n} & x
\end{array}\right]\left\{\begin{array}{c}
\left(T_{s}\right)_{1} \\
\left(T_{s}\right)_{2} \\
\left(T_{s}\right)_{3} \\
\vdots \\
\left(T_{s}\right)_{n} \\
T_{b}
\end{array}\right\}=\left\{\begin{array}{c}
y_{1} \\
y_{2} \\
y_{3} \\
\vdots \\
y_{n} \\
z
\end{array}\right\}
$$

where

$$
\begin{aligned}
& v_{i j}=\sum_{j=1}^{n} C_{i j}\left(G_{e q}\right)_{i j}-\left[\frac{\alpha I^{2} R_{l} \frac{2 \pi k_{w}}{\ln \left(r_{s} / r_{c}\right)}}{\frac{2 \pi k_{w}}{\ln \left(r_{s} / r_{c}\right)}-\alpha I^{2} R_{l}}\right]_{i} \\
& w_{i j}=-C_{i j}\left(G_{e q}\right)_{i j} \\
& x=\sum_{j=1}^{n} C_{b j}\left(G_{i n t}\right)_{b j}+2 \pi r_{b}\left[h+\left(G_{r a d}\right)_{b e}\right] \\
& y_{i}=\left\{I^{2} R_{l}\left[\left(1-\alpha T_{0}\right)+\alpha\left(\frac{\alpha I^{2} R_{l} T_{0}}{\alpha I^{2} R_{l}-\frac{2 \pi k_{w}}{\ln \left(r_{s} / r_{c}\right)}}\right)\right]\right\}_{i} \\
& z=2 \pi r_{b}\left[h+\left(G_{r a d}\right)_{b e}\right] T_{e}
\end{aligned}
$$

and the subscripts $b$ and $e$ refer to the wire bundle outer jacket surface and the environment, respectively.

Solution of the system of equations is performed by assuming initial values for each element temperature. The linearized temperature terms are formed using temperatures calculated from the previous iteration and the system is solved for wire insulation jacket surface temperatures by matrix inversion. Temperatures are computed iteratively and compared with temperatures from the previous iteration until a desired residual is attained. Finally, conductor temperatures are obtained by applying eqn (21).

\section{Comparison of the wire bundle thermal model with test data}

Development of the wire bundle thermal model was performed in conjunction with testing in an ambient environment. A wire bundle composed of $n=19$ wire elements plus an outer insulation jacket was used as the test configuration and is depicted in fig. 1. 


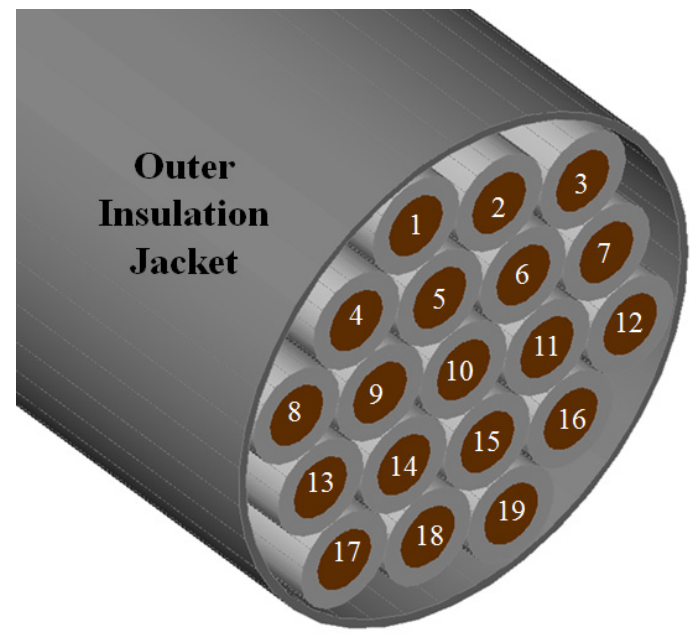

Figure 1: Wire bundle analysis configuration schematic.

Seventeen of the 19 elements were 22 AWG conductors, each jacketed in a PTFE insulation jacket with an assumed $\varepsilon=0.93$ and $k_{w}=0.238 \mathrm{~W} / \mathrm{m} \mathrm{K}$. The remaining two elements (\#6 and \#15) were fiber optic elements and, as such, carried no current. The outer jacket had an assumed emissivity, $\varepsilon=0.04$, typical for vapor deposited aluminum with an outer bundle radius, $r_{b}=0.00287 \mathrm{~m}$. Seven ambient test cases were used to demonstrate the thermal model performance, each with a background current (i.e., current in all conductors with the exception of the conductors carrying the high current, "smart short") of approximately $2.5 \mathrm{~A}$. Two conductors in the bundle (\#5 and \#10) were assumed to carry a variety of currents representing a different "smart short" scenarios (i.e., high currents beyond normal loading but too low to cause fuse or circuit breaker action). Wire conductor temperatures resulting from the various test conditions were determined through resistance measurements and computed with knowledge of the resistance at a reference temperature $\left(20^{\circ} \mathrm{C}\right)$ and the assumed temperature coefficient of resistivity, $\alpha=0.00342 / K$. Ambient temperature for the tests was $22.8^{\circ} \mathrm{C}$. All test conditions were run to steady state. Wire-to-wire and wire-tobundle jacket interface conductance, on a per unit length basis, were assumed to $0.5 \mathrm{~W} / \mathrm{m} \mathrm{K}$ and $1.0 \mathrm{~W} / \mathrm{m} \mathrm{K}$, respectively, and determined through correlation to a single test case and assumed constant over the range of cases tested. Results of the analysis as compared to test data are presented in table 1 . Note that all calculations were performed using absolute temperature with results expressed in ${ }^{\circ} \mathrm{C}$. 
Table 1: Comparison of wire bundle thermal model to ambient test data.

\begin{tabular}{|c|c|c|c|c|}
\hline $\begin{array}{c}\text { "Smart } \\
\text { Short" } \\
\text { current } \\
(\mathrm{A})\end{array}$ & $\begin{array}{c}\text { Background } \\
\text { current } \\
(\mathrm{A})\end{array}$ & $\begin{array}{c}\text { Test derived } \\
\left(T_{\text {test }}\right) \text { "Smart } \\
\text { Short" wire } \\
\text { temperature } \\
\left({ }^{\circ} \mathrm{C}\right)\end{array}$ & $\begin{array}{c}\text { Predicted } \\
\left(T_{\text {predicted }}\right) \\
\text { "Smart Short" } \\
\text { wire temperature } \\
\left({ }^{\circ} \mathrm{C}\right)\end{array}$ & $\begin{array}{c}T_{\text {test }} \\
-T_{\text {predicted }} \\
\left({ }^{\circ} \mathrm{C}\right)\end{array}$ \\
\hline 0.00 & 2.51 & 45.8 & 44.7 & 1.1 \\
\hline 4.40 & 2.51 & 54.5 & 53.7 & 0.8 \\
\hline 6.66 & 2.52 & 65.4 & 65.5 & -0.1 \\
\hline 8.49 & 2.52 & 77.1 & 79.0 & -1.9 \\
\hline 13.37 & 2.52 & 134.5 & 135.1 & -0.6 \\
\hline 16.33 & 2.52 & 188.0 & 189.2 & -1.2 \\
\hline 16.88 & 2.52 & 200.0 & 201.6 & -1.6 \\
\hline
\end{tabular}

\section{Monte Carlo analysis}

The previously discussed analysis assumed perfect knowledge of the parameters of interest. However, many of the parameters used in the analysis may not be known with a high degree of certainty. It is often of interest to the analyst to understand the sensitivity of the solution to uncertainties in one or more of the analysis parameters. To address this, a Monte Carlo analysis capability was added to the spreadsheet tool. As a demonstration of capability, a Monte Carlo analysis was performed using the parameter variations for three key variables specified in table 2 .

Table 2: $\quad$ Monte Carlo analysis parameters.

\begin{tabular}{|c|c|c|}
\hline Parameter & $\begin{array}{c}\text { Variation } \\
(+/-\%)\end{array}$ & $\begin{array}{c}\text { Distribution } \\
\text { Type }\end{array}$ \\
\hline$\alpha$ & 10 & Normal \\
\hline$G_{\text {int }}$ & 20 & Normal \\
\hline$h$ & 10 & Normal \\
\hline
\end{tabular}

As a demonstration of capability, one hundred cases were analyzed for the 13.37 A "smart short" current in conductors \#5 and \#10 with a background current of $2.52 \mathrm{~A}$ for all other conductors except for \#6 and \#15 which had no current. Individual wire conductor temperature variation, presented in ${ }^{\circ} \mathrm{C}$ was tallied and an assumed normal distribution was fitted with knowledge of the mean and standard deviation tallied for each wire and presented in fig. 2 .

In this analysis case, conductors \#5 and \#10 were energized with the smart short current and all other conductors, except \#6 and \#15, were energized with a background current of $2.52 \mathrm{~A}$. As can be seen in fig. 2, uncertainty in key analysis parameters can lead to large uncertainties in temperature predictions and may require application of considerable temperature margins for design applications. 


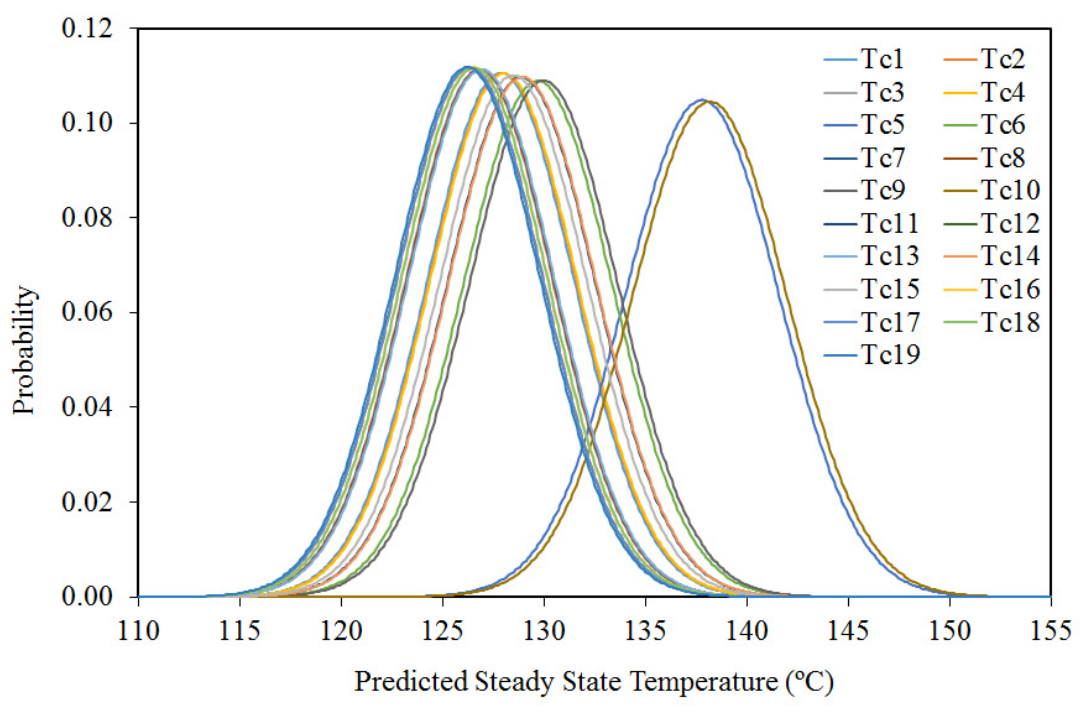

Figure 2: Temperature distributions resulting from a Monte Carlo analysis.

\section{Concluding remarks}

An analytical approach for wire bundle thermal analysis has been presented. The governing heat transfer equations have been derived. Implementation of the system of equations for solution of the wire bundle problem has been demonstrated and a means for assessing the sensitivity due to uncertainty in key analysis parameters has been investigated. Future efforts will focus on refinement of the technique and application to more complex bundle configurations (e.g., bundles composed of sub-bundles). Once completed, it is envisioned that the implementation of such models may be useful in assessing wire bundle ampacity. In practice, the bundle of interest would be modeled for the conditions of interest. A Monte Carlo analysis would be performed to assess the sensitivity of the solution to uncertainty in a variety of model parameters. A successful design is attained when the predicted wire and insulation temperatures plus margins associated with parameter uncertainties are below the wire temperature limits.

\section{Nomenclature}

$A_{s} \quad$ wire surface area $\left(\mathrm{m}^{2}\right)$;

$B_{i j} \quad$ thermal radiation interchange factor between wires $i$ and $j$;

$C_{i j} \quad$ Factor to specify connectivity between wires $i$ and $j$;

$c_{p} \quad$ air specific heat $(J / k g K)$;

$f_{h} \quad$ convective heat transfer scaling factor;

$g$ acceleration due to gravity at Earth's surface $\left(9.8 \mathrm{~m} / \mathrm{s}^{2}\right)$; 


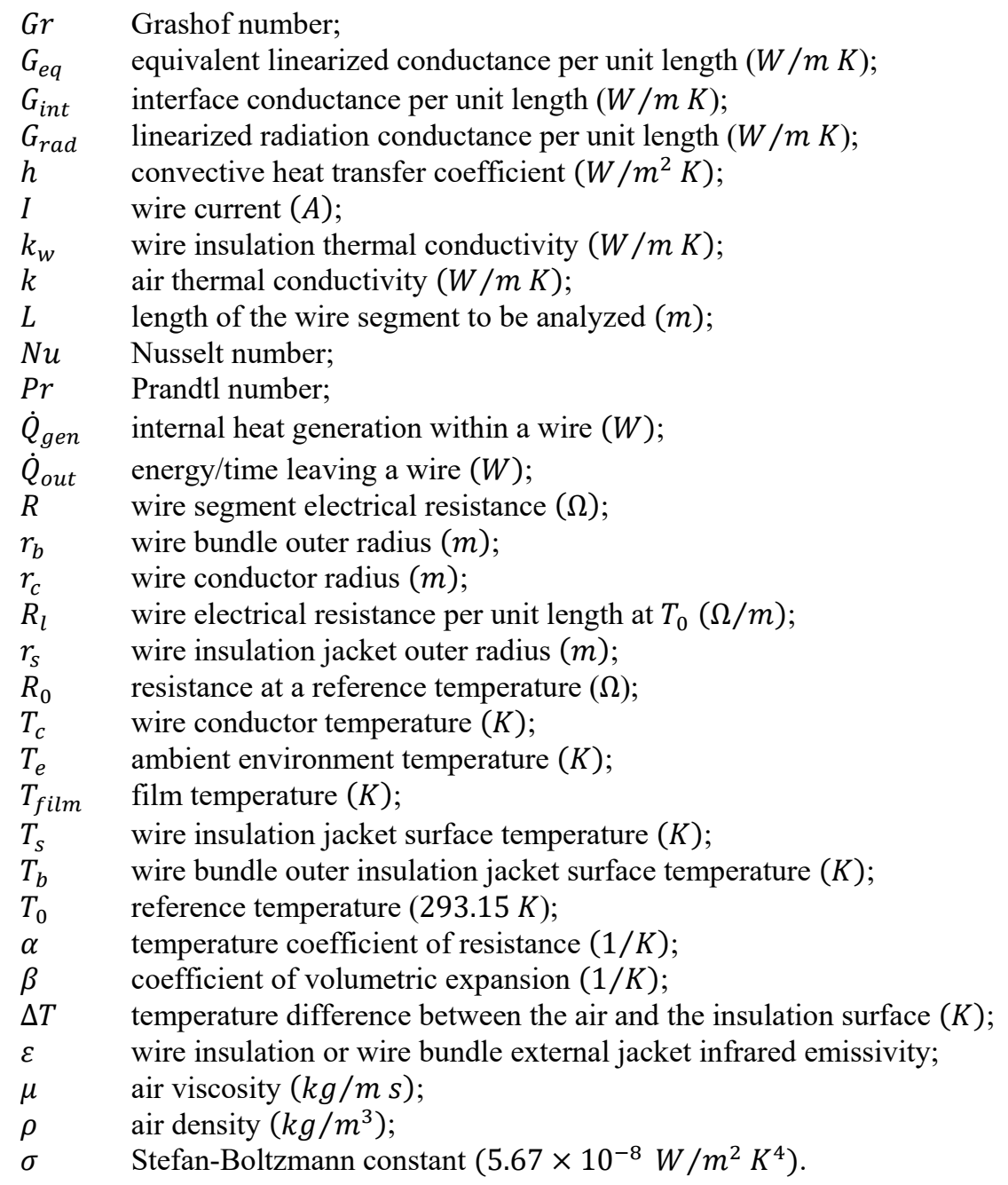

\section{Acknowledgements}

The work described in this paper was performed with the sponsorship of the NASA Engineering and Safety Center under the technical leadership of Dr. Robert Kichak of AS and D, Inc. The authors acknowledge the test support provided by Mr. Thad Johnson, Mr. Lawrence Ludwig, Mrs. Tamara Dodge, Mr. Dan Ciarlariello and Mr. Lawrence Batterson of the NASA - Kennedy Space Center.

\section{References}

[1] Cornelius, R.S., A History of the Development of New Current Ratings for Aerospace Wire, RayChem Report, circa 1978. 
[2] Wire Size Determination for Aerospace Applications, Report No. 88-220, Eagle Engineering/LMESCO under Contract to NASA (NAS-17900), December 1, 1988.

[3] Wire/Cable Selection, Report No. 2ITA017, McDonnell Douglas Space Systems Company, May 10, 1990.

[4] van Benthem, R.C., de Grave, W., Doctor, F., Taylor, S.,Nuyten, K., \& Dit Routier, P.A.J., Thermal Analysis of Wiring Bundles for Weight Reduction and Improved Safety, AIAA 2011-5111, National Aerospace Laboratory (NLR), Netherlands, International Conference on Environmental Systems, July 2011.

[5] A. Ilgevičius, H. D. Liess, Thermal Analysis of Electrical Wires by Finite Volume Method, ISSN 1392 - 1215 Elektronika ir Elektrotechnika. 2003. 4(46).

[6] Holman, J.P., Heat Transfer, $5^{\text {th }}$ Edition, McGraw-Hill Book Company: New York, pp. 274-277, 1981. 